\title{
Emotional Regulation Questionnaire (ERQ): Indicadores Psicométricos e Relações com Medidas Afetivas em Amostra Idosa
}

\author{
Emotional Regulation Questionnaire (ERQ): Psychometric Indicators \\ and Affective Relations in an Elderly Sample
}

\author{
Samila Sathler Tavares Batistoni, Tiago Nascimento Ordonez, Thaís Bento Lima da Silva*, \\ Priscila Pascarelli Pedrico do Nascimento \& Meire Cachioni \\ Universidade de São Paulo, São Paulo, Brasil
}

\begin{abstract}
Resumo
Examinou-se indicadores psicométricos do Questionário de Regulação Emocional (QRE) e suas relações com medidas de experiência afetiva, satisfação com a vida e depressão em idosos. Cento e cinquenta e três idosos $(M=66,8$ anos; $D P= \pm 5,20,71,2 \%$ feminino $)$ de uma Universidade Aberta à Terceira Idade responderam ao QRE, ao Positive and Negative Affect Schedule (PANAS) e à escala satisfação com a vida e de depressão (GDS-15). Dois fatores (Reavaliação Cognitiva; Supressão Emocional) explicaram 50,1\% da variância e os índices de consistência interna e de estabilidade temporal foram acima de 0,70 . Houve relações positivas entre Reavaliação Cognitiva, satisfação com a vida e afetos positivos e relações negativas com depressão. Maior uso da Reavaliação Cognitiva é indicativo de saúde emocional em idosos.

Palavras-chave: Regulação emocional, afetos, satisfação com a vida, depressão, idosos.
\end{abstract}

\begin{abstract}
We examined psychometric indicators of the Emotional Regulation Questionnaire (ERQ) and their relationship with measures of affective experience, satisfaction with life and depression in the elderly. One hundred and fifty-three elderly people $(M=66.8$ years, $S D=5.20,71.2 \%$ female) of an Open University for Seniors responded to the ERQ, the Positive and Negative Affect Schedule (PANAS), the Life Satisfaction Scale and the Geriatric Depression Scale (GDS-15). Two factors (Cognitive Reappraisal and Emotional Suppression) explained $50.1 \%$ of variance; internal consistency and temporal stability were above .70 . There were positive relations between Cognitive Reappraisal and life satisfaction and positive affect; and there were negative relations with depression. Increased use of Cognitive Reappraisal is an indication of emotional health in the elderly.

Keywords: Emotion regulation, affect, life satisfaction, depression, the elderly.
\end{abstract}

Teorias life-span em psicologia têm apontado a saliência das emoções como recurso adaptativo às mudanças do envelhecimento. Teorias motivacionais-emocionais propõem ganhos relacionados ao aumento da idade em metas e estratégias emocionais que visam otimizar o bemestar (Carstensen, Isaacowitz, \& Charles, 1999; Kennedy, Mather, \& Carstensen, 2004). Anteriormente descrito como um fenômeno paradoxal ("paradoxo do bem-estar na velhice"; Lawton, Kleban, Rajagopal, \& Dean 1992), os altos níveis de bem-estar relatados por idosos, mesmo

\footnotetext{
* Endereço para correspondência: Escola de Artes, Ciências e Humanidades, Universidade de São Pau1o, Avenida Arlindo Béttio, 1000, Ermelino Matarazzo, São Paulo, SP, Brasil 03828-000. E-mail: samilabatistoni@gmail.com, tiagordonez@gmail.com, gerontologathais@gmail.com, priscila.pascarelli@hotmail.com e meirec@usp.br
}

no contexto de perdas e declínios normativos, atualmente tem sido interpretados à luz da interseção entre emoções e cognição nos processos adaptativos de seleção, otimização e compensação de déficits.

O estudo de Lawton e colaboradores (1992) foi um dos primeiros a revelar que idosos diferiam dos mais jovens quanto às habilidades de influenciar a ocorrência e o conteúdo das emoções. Após esses primeiros estudos, uma série de outros evidenciam que idosos são mais propensos a relatar maior controle emocional, principalmente regular a experiência interna de estados negativos como a raiva e da expressão externa de felicidade e tristeza (Blanchard-Fields, Stein, \& T. L. Watson, 2004; Gross et al., 1997).

Scheibe e Carstensen (2010), em artigo de revisão, apontaram evidências da interseção entre emoções e cognição por meio do chamado "efeito de positividade" (na atenção, memória e tomada de decisões) baseando-se 
na premissa de que os idosos tornam-se mais sensíveis a informação de conteúdo emocional positivo e menos sensíveis ou avessos à informação de conteúdo emocional negativo. Em conformidade com esses achados, autores como Urry e Gross (2010) têm proposto que os idosos selecionam e otimizam estratégias de regulação emocional como um reflexo dos recursos adaptativos disponíveis sendo relevante investigar as relações entre regulação emocional e envelhecimento.

Regulação emocional, segundo Gross e colaboradores (Ehring, Tuschen-Caffier, Schunulle, Fischer, \& Gross, 2010; Gross, 1998a; Gross \& John, 2003) diz respeito a ativação de processos pelos quais os indivíduos influenciam quais emoções irão ter, quando irão tê-las e como irão experienciar e expressar essas emoções. Os processos de regulação podem ser automáticos ou controlados, conscientes ou inconscientes e podem ter efeitos em um ou mais pontos do processo de geração de uma emoção (Gross, 1998b). Assim, ao longo do contínuo entre a percepção de um estímulo ou contexto emocional e a expressão fisiológica e comportamental da emoção, cinco conjuntos de estratégias podem ser selecionadas e empregadas no manejo das emoções. Esses cinco conjuntos podem ser agrupados em estratégias focadas nos antecedentes (seleção da situação, modificação da situação, mudança do foco atencional, reavaliação cognitiva) ou focadas na regulação das respostas emocionais (supressão das respostas emocionais).

Segundo John e Gross (2004), a maioria dos estudos disponíveis examinam apenas duas estratégias representativas desses processos, ou seja, a reavaliação cognitiva (uma forma de mudança cognitiva que envolve a reinterpretação da situação emocional de forma a altera o impacto emocional da mesma) e a supressão das respostas emocionais (modulação da resposta emocional que envolve inibição dos comportamentos de expressão emocional), uma vez que estas encontram ressonância nos estudos baseados no paradigma clássico do estresse e coping (Lazarus \& Folkman, 1984). Porém, estratégias de regulação emocional são mais amplas que estratégias de coping, uma vez que as primeiras não visam apenas lidar com emoções negativas, mas envolve amplificar, manter ou diminuir estados emocionais positivos ou negativos (Gross, 1998a).

Para examinar diferenças individuais no uso sistemático dessas estratégias, Gross e John (2003) propuseram o instrumento de autorrelato, o Emotion Regulation Questionnaire (ERQ). Esta medida envolve 10 itens referentes aos dois processos de regulação emocional (6 itens compõem o fator "reavaliação cognitiva" e quatro itens compõem o fator "supressão emocional") e tem sido utilizada em diversos estudos internacionais (S. Gillanders, Wild, Deighan, \& D. Gillanders, 2008; John \& Gross, 2007; Tamir, John, Srivastava, \& Gross, 2007).

Estudos revelam que estes dois conjuntos de estratégias de regulação emocional relacionam-se diferentemente com medidas de adaptação, como bem-estar emocional e saúde física. A estratégia de reavaliação tem sido associada com eficácia na diminuição de desgosto (Gross, 1998a), maior experiência e expressão de emoções positivas, satisfação com a vida e menos sintomas depressivos (Gross \& John, 2003; John \& Gross, 2004) e a estratégia de supressão, por sua vez, relaciona-se com aumento da atividade simpática (Gross, 1998a), comprometimento da memória para informações sociais (Richards \& Gross, 2000) e mais fortemente com medidas de psicopatologia como ansiedade e depressão (Gross \& John, 2003) e senso de inautenticidade (Sheldon, Ryan, Rawsthorne, \& Ilardi, 1997).

John e Gross (2004) compararam três grupos etários e também demostraram que idosos utilizaram menos a estratégia de supressão e revelaram maiores índices de bem-estar. Nesse estudo, houve diferenças de gênero indicando que mulheres idosas em relação às mulheres jovens diminuíram consideravelmente a utilização de supressão e demostraram aumento em reavaliação. Entre idosos, tal escala tem identificado que o uso da reavaliação cognitiva resulta por exemplo, em mais satisfação com o trabalho entre idosos não aposentados (Yahyagil \& Ýkier, 2009).

Para uso entre a população adulta brasileira, Boian, Soares e Silva (2009), realizaram uma adaptação linguística dessa medida e a disponibilizaram no site do Stanford Psychophysiology Laboratory (coordenado pelo professor James Gross). Porém, não identificou-se na literatura nacional a publicação em revistas científicas qualificadas o exame psicométrico do questionário e também indicadores de validade e fidedignidade deste instrumento quando aplicado a idosos. Considera-se o exame desses indicadores necessário, uma vez que tal instrumento pode ser útil na identificação e elucidação das relações entre estratégias de regulação emocional e o bem-estar de idosos, lacunas também identificadas na literatura de pesquisa nacional.

Nesse sentido, o presente estudo visou alcançar dois objetivos principais: (a) Derivar indicadores psicométricos de validade e fidedignidade (validade de construto, consistência interna e confiabilidade teste-reteste) da versão brasileira da escala de regulação emocional aplicada a idosos; (b) Identificar relações entre as estratégias de regulação emocional (reavaliação cognitiva e supressão emocional) e medidas de experiência afetiva, satisfação com a vida e depressão entre idosos.

\section{Método}

\section{Procedimentos}

O presente estudo faz parte da pesquisa "Educação Permanente - Benefícios da Universidade Aberta à Terceira Idade da Escola de Artes, Ciências e Humanidades da Universidade de São Paulo - EACH USP", que conta com o financiamento do Instituto Nacional de Estudos e 
Pesquisas Educacionais Anísio Teixeira - Ministério da Educação (Edital de Seleção no 02/2009 - INEP/MEC). Trata-se de um estudo prospectivo visando identificar as características e os benefícios da participação de idosos em uma Universidade Aberta à Terceira idade, aprovado pelo Comitê de Ética em Pesquisa do Instituto de Psicologia da USP, parecer de número 2010.043.

O estudo foi realizado no início e no final de dois semestres de atividades, com medidas de base no momento da matrícula dos idosos nas atividades e uma medida prospectiva ao final do semestre. Ao realizar a matrícula, os idosos foram convidados a participar da pesquisa. Nesta ocasião, foram explicados os objetivos do estudo e os procedimentos da coleta de dados. Os que aceitaram participar do estudo foram entrevistados no mesmo dia ou em data conveniente para os mesmos. Esta entrevista foi realizada por graduandos do curso de Gerontologia da EACH previamente treinados para esta atividade. O tempo médio de duração da entrevista foi 90 minutos.

Para o presente estudo, trabalhou-se com os dados coletados no início do primeiro semestre de pesquisa, selecionando-se do protocolo as variáveis de interesse. $\mathrm{O}$ estudo também analisou dados de reteste de uma subamostra ( $42 \%$ da amostra) randomicamente sorteada entrevistada 20 dias após a medida de base, cujo protocolo continha, entre outros, o instrumento alvo do presente estudo (QRE) a fim de identificar a confiabilidade teste-teste do mesmo. Apenas um idoso recusouse a participar do reteste.

\section{Amostra}

Participaram do presente estudo 153 idosos com idade igual e superior a sessenta anos, inscritos no $1^{\circ}$ semestre de 2010 na Universidade Aberta à Terceira Idade (UnATI EACH-USP). Destes, 63 foram submetidos à retestagem.

\section{Instrumentos}

Selecionou-se do protocolo as seguintes medidas:

Variáveis Sociodemográficas: Gênero, idade, escolaridade (por anos de estudo), renda (por faixas salariais) e estado civil.

Questionário de Regulação Emocional - QRE (Emotion Regulation Questionnaire - ERQ; Gross \& John, 2003), que consiste numa medida de autorrelato de 10 itens a respeito de duas estratégias comuns pelas quais as pessoas podem regular as emoções: 4 itens medem supressão das emoções e 6 medem o uso da estratégia de reavaliação das emoções. As respostas aos itens são respondidas numa escala Likert que varia de 1 (Discordo totalmente) a 7 (Concordo totalmente). Escores mais altos indicam o uso mais freqüente de uma determinada estratégia. Originalmente, o questionário apresenta boas propriedades com coeficiente alfa de Cronbach's entre
0,79 (para a subscala de reavaliação cognitiva) e 0,73 (para a subscala de supressão Emocional). Confiabilidade teste-reteste de 0,69 para ambas subscalas e análise fatorial (rotação ortogonal) sustentando uma estrutura de dois fatores. Para o presente estudo, utilizamos a versão brasileira de Boian et al. (2009).

Afetos Positivos e Negativos: medido através da Escala de Ânimo Positivo e Negativo (EAPN; Siqueira, Martins, \& Moura, 1999), versão brasileira do PANAS (Watson, Clark, \& Tellegen, 1988). Em uma escala que varia de 1 (pouco) a 5 (extremamente), solicita-se ao sujeito que avalie quão frequentemente, ao longo da última semana, ele experimentou afetos positivos (feliz, alegre, animado, bem, satisfeito, contente) e negativos (irritado, desmotivado, angustiado, deprimido, chateado, nervoso, triste, desanimado).

Escala para Medida da Satisfação Geral com a Vida: escala de item único que solicita ao indivíduo que atribua uma nota de 1 a 10 apresentada graficamente $(1=$ a pior vida e $10=$ a melhor vida) à sua satisfação atual com a vida (Freire, 2001; Neri, 2002; Santos, 2003). A referida escala é uma forma modificada da Ladder Scale criada por Cantril (1967, citado por Freire, 2001).

Escala de Depressão Geriátrica (GDS-15): A Geriatric Depression Scale (Yesavage et al., 1983) validada para os idosos brasileiros por O. P. Almeida e S. A. Almeida (1999) visa identificar presença significativa de sintomas depressivos. Composta por 15 perguntas com respostas dicotômicas (sim x não - presença ou ausência do sintoma), fornece como parâmetro para o rastreio de depressão o escore igual ou maior que 6 pontos (Frank \& Rodrigues, 2006).

\section{Análise dos Dados}

Para alcançar o primeiro objetivo deste estudo, os dados relativos às respostas dos 153 idosos ao Questionário de Regulação Emocional (QRE) foram submetidos à Análise Fatorial Exploratória e derivado o coeficiente $\alpha$ de Cronbach. A estabilidade ou reprodutibilidade do Questionário de Regulação Emocional foi testada através da comparação dos escores obtidos da QRE entre duas aplicações (Teste e Reteste). A reprodutibilidade interobservador foi avaliada pelo coeficiente de correlação intraclasse de duas vias, efeitos mistos, com concordância absoluta (two-way mixed intraclass correlation coefficient with absolute agreement).

Para as análises referentes ao segundo objetivo deste estudo foram feitas tabelas com estatísticas descritivas do Questionário de Regulação Emocional, com medidas de posição e dispersão. Em seguida, compararam-se os escores do questionário entre os sexos, utilizando-se o teste $U$ de Mann-Whitney, devido à ausência de distribuição normal da variável dependente em questão. Para analisar a relação entre os fatores do Questionário de Regulação Emocional e as variáveis numéricas, como 
Batistoni, S. S. T., Ordonez, T. N., Silva, T. B. L., Nascimento, P. P. P. \& Cachioni, M. (2013). Emotional Regulation Questionnaire (ERQ): Indicadores Psicométricos e Relações com Medidas Afetivas em Amostra Idosa.

idade, escolaridade e renda e os relativos às Escalas de Satisfação com a Vida, de Estado de Ânimo (Afetos positivos e Afetos Negativos) e de Depressão Geriátrica também foram submetidos à correlação de Spearman para se analisar possíveis correlações entre estas variáveis e o Questionário de Regulação Emocional.

As análises foram realizadas por meio do programa de estatística computacional: Statistica v. 7.0 (2004). O nível de significância adotado para os testes estatísticos foi de $5 \%$, ou seja, $p$-valor $<0,05$.

\section{Resultados}

\section{Caracterização da Amostra}

A Tabela 1 traz as características sociodemográficas da amostra, composta, em sua maioria, pelo sexo feminino e por idosos casados. A média de idade dos participantes foi de 66,79 anos $( \pm 5,20)$ e de escolaridade de média de $10,84( \pm 8,3)$ anos. A Tabela 1 também revela que apenas quanto ao estado civil a subamostra de reteste $(n=63)$ diferenciou-se da amostra total devido ao maior número de indivíduos divorciados, separados ou desquitados.

Tabela 1

Características Sociodemográficas da Amostra Total (N=153) e da Subamostra do Reteste do Estudo (N=63)

\begin{tabular}{|c|c|c|c|c|c|}
\hline \multirow[b]{2}{*}{ Variáveis } & \multicolumn{2}{|c|}{ Amostra total } & \multicolumn{2}{|c|}{ Amostra do Reteste } & \multirow[b]{2}{*}{$p$-valor } \\
\hline & $N$ & $\%$ & $n$ & $\%$ & \\
\hline \multicolumn{6}{|l|}{ Sexo } \\
\hline Masculino & 44 & 28,76 & 18 & 28,57 & \\
\hline Feminino & 109 & 71,24 & 45 & 71,43 & $1,000^{\mathrm{a}}$ \\
\hline \multicolumn{6}{|l|}{ Grupos de idade } \\
\hline 60-64 & 63 & 41,18 & 25 & 39,68 & \\
\hline $65-70$ & 50 & 32,68 & 24 & 38,10 & \\
\hline 71 ou mais & 40 & 26,14 & 14 & 22,22 & $0,658^{\mathrm{b}}$ \\
\hline Média $(D P \pm)$ & 66,79 & $(5,20)$ & 66,40 & $(4,58)$ & \\
\hline Mediana & \multicolumn{2}{|c|}{65,00} & \multicolumn{2}{|c|}{66,00} & \\
\hline Mínimo - Máximo & \multicolumn{2}{|c|}{$60,00-85,00$} & \multicolumn{2}{|c|}{$60,00-78,00$} & \\
\hline \multicolumn{6}{|l|}{ Estado Civil } \\
\hline Solteiros & 18 & 11,76 & 11 & 17,46 & \\
\hline Casados/União estável & 78 & 50,98 & 37 & 58,73 & \\
\hline Divorciados, Separados, Desquitados. & 16 & 10,46 & 1 & 1,59 & \\
\hline Viúvos & 41 & 26,80 & 14 & 22,22 & $0,018^{\mathrm{a}}$ \\
\hline \multicolumn{6}{|l|}{ Escolaridade (em anos) } \\
\hline Ens. Fundamental (Até 8 anos) & 42 & 27,45 & 20 & 31,75 & \\
\hline Ens. Médio (De 9 a 11 anos) & 65 & 42,48 & 24 & 38,10 & \\
\hline Ens. Superior (12 anos ou mais) & 46 & 30,07 & 19 & 30,16 & $0,100^{\mathrm{b}}$ \\
\hline Média $(D P \pm)$ & 10,84 & $(8,31)$ & 9,47 & $(4,08)$ & \\
\hline Mediana & \multirow{2}{*}{\multicolumn{2}{|c|}{$\begin{array}{c}10,50 \\
1,00-95,00\end{array}$}} & \multirow{2}{*}{\multicolumn{2}{|c|}{$\begin{array}{c}9,50 \\
1,00-21,00\end{array}$}} & \\
\hline Mínimo - Máximo & & & & & \\
\hline \multicolumn{6}{|l|}{ Renda Familiar } \\
\hline Até $2.0 \mathrm{SM}$ & 46 & 25,00 & 15 & 23,81 & \\
\hline De 2.1 a $3.0 \mathrm{SM}$ & 33 & 17,90 & 9 & 14,29 & \\
\hline De 3.1 a $4.0 \mathrm{SM}$ & 35 & 19,00 & 15 & 23,81 & \\
\hline De 4.1 a $5.0 \mathrm{SM}$ & 25 & 13,60 & 8 & 12,70 & \\
\hline Mais de $5.1 \mathrm{SM}$ & 45 & 24,50 & 16 & 25,40 & $0,270^{\mathrm{b}}$ \\
\hline
\end{tabular}

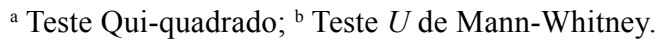

Indicadores de Validade de Construto do QRE

Os testes Esfericidade de Bartlett e o de Kaiser-MeyerOlkin indicaram respectivamente que houve correlação entre os itens avaliados do questionário de regulação emocional $\left[\chi^{2}(g l=45)=352,804 ; p<0,001\right]$ e adequação da amostra $(\mathrm{KMO}=0,764)$ para realização de análise fatorial exploratória. As análises de Scree Plot e a de aná- lise fatorial exploratória (rotação Varimax) do Questionário de Regulação Emocional (QRE) indicaram a existência de dois fatores com autovalores de 3,22, e 1,79 que juntos explicaram $50,1 \%$ da variância total.

A Tabela 2 apresenta as cargas fatoriais dos itens que compuseram cada um dos fatores, cujo critério de pertencimento ao fator foi possuir carga maior que 0,50 . 
Tabela 2

Cargas Fatoriais dos Itens que Compuseram os Dois Fatores do QRE

Cargas Fatoriais

Descrição do item

Fator 1 Fator 2

1. Quando quero sentir mais emoções positivas (como alegria ou contentamento), mudo o que estou pensando.

2. Eu conservo as minhas emoções para mim.

3. Quando quero sentir menos emoções negativas (como tristeza ou raiva) mudo o que estou pensando.

4. Quando estou sentindo emoções positivas, tenho cuidado para não expressá-las.

5. Quando estou perante uma situação estressante, procuro pensar de uma forma que me ajude a ficar calmo.

6. Eu controlo as minhas emoções não as expressando.

$0,52-0,02$

$0,19 \quad 0,73$

$0,67 \quad 0,07$

$-0,16 \quad 0,72$

$0,62 \quad 0,22$

$0,05 \quad 0,83$

7. Quando quero sentir mais emoções positivas, eu mudo o que estou pensando em relação à situação.

$0,69 \quad 0,06$

8. Eu controlo as minhas emoções modificando a forma de pensar sobre a situação em que me encontro.

$0,70 \quad 0,02$

$0,44 \quad 0,55$

9. Quando estou sentindo emoções negativas, tento não expressá-las.

10. Quando eu quero sentir menos emoções negativas, mudo à forma como estou pensando em relação à situação.

$0,76 \quad 0,07$

Nota. Método de extração: Análise de componentes principais, rotação Varimax.

Os seis itens que compuseram o primeiro fator referiram-se às estratégias de regulação emocional do tipo "Reavaliação Cognitiva". Os itens 8 e 10 do instrumento obtiveram as maiores cargas. $\mathrm{O}$ segundo fator foi composto por 4 itens que referiram-se a estratégias do tipo "supressão emocional". É possível identificar que todos os valores, com exceção do item 9, estão acima de 0,70 .
Consistência Interna do QRE

A Tabela 3 apresenta os coeficientes de consistência interna ( $\alpha$ de Cronbach) e de correlação teste-reteste do Questionário de Regulação Emocional. Observa-se que o instrumento como um todo e cada fator alcançou consistência moderada $(>0,60)$ e a retirada dos itens com as menores cargas fatoriais não revelou-se interessante, pois resulta em diminuição dos coeficientes.

Tabela 3

Indicadores de Consistência Interna (coeficientes á de Cronbach) e de Correlação Teste-Reteste do Questionário de Regulação Emocional

\begin{tabular}{lccccccc}
\hline $\begin{array}{l}\text { Escala Total } \\
\text { e Fatores da ERE }\end{array}$ & $\begin{array}{c}\mathrm{n}^{\circ} \mathrm{de} \\
\text { itens }\end{array}$ & $\alpha$ & $\begin{array}{c}\text { Coeficientes } \\
\text { baseados em itens } \\
\text { padronizados }\end{array}$ & $\begin{array}{c}\text { Item } \\
\text { com menor } \\
\text { consistência }\end{array}$ & $\begin{array}{c}\text { Correlação } \\
\text { com } \\
\text { o total }\end{array}$ & $\begin{array}{c}\text { Coeficiente } \\
\text { (após a retirada } \\
\text { dos itens }\end{array}$ & $\begin{array}{c}\text { Correlação } \\
\text { teste-reteste } \\
(p<0,001)\end{array}$ \\
\hline Escore Total & 10 & 0,730 & 0,752 & 4 & 0,211 & 0,748 & 0,706 \\
Fator 1- Reavaliação & 6 & 0,749 & 0,758 & 1 & 0,367 & 0,754 & 0,752 \\
Fator 2-Supressão & 4 & 0,690 & 0,700 & 4 & 0,260 & 0,694 & 0,736 \\
\hline
\end{tabular}

\section{Estabilidade Temporal do QRE}

A reprodutibilidade interobservador foi avaliada pelo coeficiente de correlação intraclasse de duas vias, efeitos mistos, com concordância absoluta (two-way mixed intraclass correlation coefficient with absolute agreement) e seu IC de $95 \%$. Os dois fatores do questionário, assim como o seu escore total, apresentaram um coeficiente de correlação teste-reteste superior a 0,70 (Tabela 3 ), indicador de boa estabilidade entre os dois momentos da avaliação (Hripcsak \& Heitjan, 2002).
Relações entre Regulação Emocional, Afetos Positivos e Negativos, Satisfação com a Vida e Depressão

A Tabela 4 descreve a frequência e as medidas de posição e dispersão das variáveis de regulação emocional, experiência afetiva, satisfação com a vida e depressão. A pontuação média da amostra no fator de reavaliação cognitiva foi maior do que a média em supressão emocional. A frequência de afetos positivos foi superior à experiência de afetos negativos. A distribuição dos tercis 
Batistoni, S. S. T., Ordonez, T. N., Silva, T. B. L., Nascimento, P. P. P. \& Cachioni, M. (2013). Emotional Regulation Questionnaire (ERQ): Indicadores Psicométricos e Relações com Medidas Afetivas em Amostra Idosa.

Tabela 4

Medidas de Distribuição, Posição e Dispersão da Amostra para as Variáveis de Regulação Emocional, Experiência Afetiva, Satisfação com a Vida e Depressão

\begin{tabular}{|c|c|c|c|c|c|c|}
\hline & $n(\%)$ & $M$ & $D P \pm$ & Mínimo & Mediana & Máximo \\
\hline \multicolumn{7}{|c|}{ Questionário de Regulação Emocional } \\
\hline Reavaliação Cognitiva & $153(100)$ & 5,53 & 0,89 & 2,83 & 5,67 & 7,00 \\
\hline Supressão Emocional & $153(100)$ & 4,40 & 1,36 & 1,00 & 4,50 & 7,00 \\
\hline \multicolumn{7}{|l|}{ Afetos } \\
\hline Positivos & $153(100)$ & 3,75 & 0,66 & 1,20 & 3,80 & 5,00 \\
\hline Negativos & $153(100)$ & 1,77 & 0,64 & 1,00 & 1,60 & 3,90 \\
\hline \multicolumn{7}{|l|}{ Satisfação com a Vida } \\
\hline Tercil $1(\leq 7)$ & $46(30)$ & 6,24 & 0,92 & 4,00 & 6,50 & 7,00 \\
\hline Tercil $2(=8)$ & $45(29,4)$ & 8,00 & 0,00 & 8,00 & 8,00 & 8,00 \\
\hline Tercil $3(\geq 9)$ & $62(40,6)$ & 9,52 & 0,50 & 9,00 & 10,00 & 10,00 \\
\hline Escore Total & $153(100)$ & 8,08 & 1,49 & 4,00 & 8,00 & 10,00 \\
\hline \multicolumn{7}{|l|}{ Sintomas Depressivos } \\
\hline $\operatorname{Sim}(\mathrm{GDS} \geq 6)$ & $13(8,5)$ & 7,46 & 1,51 & 6,00 & 7,00 & 10,00 \\
\hline Não $(G D S \leq 5)$ & $140(91,5)$ & 2,19 & 1,34 & 0,00 & 2,00 & 5,00 \\
\hline Escore Total & $153(100)$ & 2,64 & 2,00 & 0,00 & 2,00 & 10,00 \\
\hline
\end{tabular}

Tabela 5

Comparações entre Características Sociodemográficas e Uso de Estratégias de Reavaliação Cognitiva e Supressão Emocional

\begin{tabular}{|c|c|c|c|c|}
\hline \multirow[b]{2}{*}{ Variáveis } & \multicolumn{2}{|c|}{ Reavaliação } & \multicolumn{2}{|c|}{ Supressão } \\
\hline & $M$ & $D P \pm$ & $M$ & $D P \pm$ \\
\hline \multicolumn{5}{|l|}{ Sexo } \\
\hline Masculino & 5,00 & 0,66 & 4,70 & 0,94 \\
\hline Feminino & 6,00 & 0,96 & 4,28 & 1,48 \\
\hline$p$-valor ${ }^{\mathrm{a}}$ & \multicolumn{2}{|c|}{0,109} & \multicolumn{2}{|c|}{0,162} \\
\hline \multicolumn{5}{|l|}{ Grupos de idade } \\
\hline $60-64$ & 5,00 & 0,86 & 4,19 & 1,34 \\
\hline $65-70$ & 6,00 & 0,85 & 4,59 & 1,30 \\
\hline 71 ou mais & 6,00 & 0,97 & 4,51 & 1,43 \\
\hline$p$-valor ${ }^{\mathrm{b}}$ & \multicolumn{2}{|c|}{0,225} & \multicolumn{2}{|c|}{0,235} \\
\hline \multicolumn{5}{|l|}{ Estado Civil } \\
\hline Solteiros & 5,00 & 0,93 & 4,99 & 1,06 \\
\hline Casados/União estável & 5,00 & 0,87 & 4,18 & 1,29 \\
\hline Divorciados, Separados, Desquitados. & 5,00 & 1,05 & 4,41 & 1,54 \\
\hline Viúvos & 6,00 & 0,78 & 4,57 & 1,46 \\
\hline$p$-valor ${ }^{\mathrm{b}}$ & \multicolumn{2}{|c|}{$0,040^{*}$} & \multicolumn{2}{|c|}{0,087} \\
\hline \multicolumn{5}{|l|}{ Escolaridade (em anos) } \\
\hline Ens. Fundamental (Até 8 anos) & 5,00 & 0,82 & 4,67 & 1,23 \\
\hline Ens. Médio (De 9 a 11 anos) & 6,00 & 0,91 & 4,32 & 1,42 \\
\hline Ens. Superior (12 anos ou mais) & 5,00 & 0,91 & 4,27 & 1,38 \\
\hline$p$-valor ${ }^{\mathrm{b}}$ & \multicolumn{2}{|c|}{0,412} & \multicolumn{2}{|c|}{0,456} \\
\hline \multicolumn{5}{|l|}{ Renda Familiar } \\
\hline Até $2.0 \mathrm{SM}$ & 6,00 & 0,89 & 4,75 & 1,32 \\
\hline De 2.1 a $3.0 \mathrm{SM}$ & 6,00 & 0,84 & 4,13 & 1,54 \\
\hline De 3.1 a $4.0 \mathrm{SM}$ & 6,00 & 0,82 & 4,65 & 1,27 \\
\hline De 4.1 a $5.0 \mathrm{SM}$ & 5,00 & 0,99 & 4,36 & 1,13 \\
\hline Mais de $5.1 \mathrm{SM}$ & 5,00 & 0,92 & 4,06 & 1,36 \\
\hline$p$-valor $^{\mathrm{b}}$ & \multicolumn{2}{|c|}{0,481} & \multicolumn{2}{|c|}{0,175} \\
\hline
\end{tabular}

${ }^{a}$ Teste U de Mann-Whitney; ${ }^{\mathrm{b}}$ Teste Kruskal-Wallis, seguido de teste de múltipla comparação (Multiple Comparisons z'values).

* Diferença estatística no teste de múltipla comparação, ser casado/ter união estável e ser viúvo $(p=0,05)$. 
basearam-se em pontuações elevadas (7,8 e 9 pontos) e a pontuação média na escala de sintomas depressivos em 15 pontos foi de 2,64 $(D P= \pm 2,0)$. Depreende-se da frequência de indivíduos que pontuaram presença significativa de sintomas depressivos a prevalência de $8,5 \%$.

A Tabela 5 apresenta as pontuações médias nas estratégias de regulação emocional entre os grupos de características sociodemográficas. Apenas a característica de ser viúvo mostrou diferença significativa com maior uso de reavaliação cognitiva.
Para análise das correlações entre todas as variáveis do estudo foram feitas análises de correlação de Spearman (Tabela 6). Observou-se, que renda e escolaridade tiveram correlação positiva significativa. Reavaliação cognitiva associou-se positivamente com supressão emocional, afetos positivos e satisfação com a vida e negativamente com afetos negativos. Afetos positivos associaram-se positivamente com satisfação com a vida e negativamente com depressão. Afetos negativos, por sua vez, associaramse positivamente depressão e negativamente com satisfação com a vida e com os afetos positivos. Satisfação com a vida associou-se negativamente com depressão.

Tabela 6

Matriz de Correlação de Spearman entre as Variáveis Sociodemográficas, Afetos Positivos, Negativos, Satisfação com a Vida, Depressão e Estratégias de Regulação Emocional

\begin{tabular}{|c|c|c|c|c|c|c|c|c|c|c|}
\hline & & 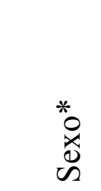 & $\frac{0}{\tilde{J}}$ & $\begin{array}{l}\frac{0}{0} \\
\frac{\tilde{J}}{0} \\
\bar{\Xi} \\
0 \\
0 \\
\text { II }\end{array}$ & $\begin{array}{l}\frac{\pi}{0} \\
\stackrel{0}{2}\end{array}$ & 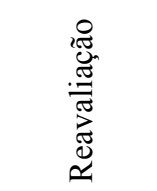 & 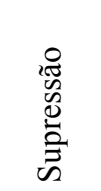 & : & $\begin{array}{l}\stackrel{n}{0} \\
\stackrel{D}{\mathbb{Z}} \\
\stackrel{0}{0} \\
Z\end{array}$ & 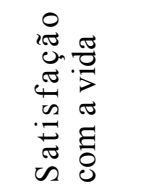 \\
\hline \multirow[t]{2}{*}{ Idade } & $r$ & $-0,05$ & & & & & & & & \\
\hline & $p$ & 0,505 & & & & & & & & \\
\hline \multirow[t]{2}{*}{ Escolaridade } & $r$ & $-0,13$ & $-0,10$ & & & & & & & \\
\hline & $p$ & 0,113 & 0,205 & & & & & & & \\
\hline \multirow[t]{2}{*}{ Renda } & $r$ & $-0,17$ & $-0,01$ & 0,33 & & & & & & \\
\hline & $p$ & 0,036 & 0,948 & $<0,001 * *$ & & & & & & \\
\hline \multirow[t]{2}{*}{ QRE- Reavaliação } & $r$ & 0,13 & 0,09 & 0,00 & $-0,10$ & & & & & \\
\hline & $p$ & 0,110 & 0,243 & 0,962 & 0,199 & & & & & \\
\hline \multirow[t]{2}{*}{ QRE- Supressão } & $r$ & $-0,11$ & 0,10 & $-0,19$ & $-0,13$ & 0,24 & & & & \\
\hline & $p$ & 0,162 & 0,198 & 0,022 & 0,101 & $0,002 * *$ & & & & \\
\hline \multirow[t]{2}{*}{ Afetos Positivos } & $r$ & $-0,20$ & 0,15 & 0,06 & 0,03 & 0,37 & 0,04 & & & \\
\hline & $p$ & 0,012 & 0,063 & 0,434 & 0,754 & $<0,001 * *$ & 0,590 & & & \\
\hline \multirow[t]{2}{*}{ Afetos Negativos } & $r$ & 0,19 & $-0,19$ & $-0,01$ & 0,00 & $-0,34$ & $-0,12$ & $-0,54$ & & \\
\hline & $p$ & 0,021 & 0,018 & 0,899 & 0,988 & $<0,001 * *$ & 0,147 & $<0,001 * *$ & & \\
\hline \multirow[t]{2}{*}{ Satisfação com a vida } & $r$ & 0,01 & 0,16 & $-0,02$ & 0,03 & 0,25 & 0,06 & 0,38 & $-0,41$ & \\
\hline & $p$ & 0,889 & 0,050 & 0,817 & 0,691 & $0,002 * *$ & 0,493 & $<0,001 * *$ & $<0,001 * *$ & \\
\hline \multirow[t]{2}{*}{ GDS } & $r$ & 0,10 & 0,04 & $-0,11$ & $-0,04$ & $-0,22$ & $-0,08$ & $-0,39$ & 0,39 & $-0,37$ \\
\hline & $p$ & 0,206 & 0,624 & 0,175 & 0,645 & 0,006 & 0,322 & $<0,001 * *$ & $<0,001 * *$ & $<0,001 * *$ \\
\hline
\end{tabular}

Nota. *Sexo: variável dicotômica, $0=$ masculino e $1=$ feminino. $* * p<0.05$.

\section{Discussão}

Em consonância com os achados de Gross e Levenson (1993), o QRE revelou indicadores válidos a respeito do construto alvo uma vez que este foi composto por dois domínios representativos das estratégias de regulação emocional, a saber reavaliação cognitva e supressão emocional, índice de consistência interna satisfatório e semelhante ao encontrado nos estudos de Gross e John (2003) com amostras jovens e de meia-idade americanas. O QRE também revelou bons índices de estabilidade temporal indicando que o instrumento também se mostra fidedigno quando aplicado a idosos brasileiros.
O índice de correlação entre o fator 1 (reavaliação) e o fator 2 (supressão) foi positivo embora de fraca intensidade. John e Gross (2004), ao também encontrarem essas associações, refutaram os modelos de regulação emocional unidimensionais e que defendem o uso exclusivo de uma ou outra estratégia pelos indivíduos. Os autores então defendem que, embora sejam estratégias independentes, refletem que os indivíduos diferem apenas em grau no uso das mesmas. Dados do presente estudo também sustentam essa concepção, uma vez que, entre idosos não revelaram a tendência de um uso exclusivo de uma ou outra estratégia, embora tenha havido maior uso da estratégia de reavaliação. 
Os resultados sobre experiência afetiva, satisfação com a vida, depressão e os derivados da aplicação do QRE entre idosos frequentadores da UnATI - EACH revelaram interessantes relações com a literatura. A amostra pesquisada revelou maior experiência de afetos positivos do que negativos, média elevada em satisfação geral com a vida e em média pontuou baixo na escala de sintomas depressivos. Entretanto, a prevalência de sintomas depressivos medida pela GDS foi inferior aos $15 \%$ encontrada entre idosos residentes na comunidade (Almeida \& Almeida, 1999), o que talvez reflita o contexto de desenvolvimento da pesquisa e composição da amostra por idosos saudáveis e ativos socialmente.

Os idosos do estudo também pontuaram em média mais alto nos itens referentes à estratégia de regulação emocional por meio da reavaliação cognitiva. A partir de uma perspectiva desenvolvimental, tal dado ganho significado, visto que o envelhecimento associa-se com aumento em experiência de vida e maior discernimento a respeito dos custos e benefícios de diferentes formas de regulação emocional, impulsionando-os a selecionar e a aplicar estratégias mais saudáveis e adaptativas. Estudos sobre diferenças individuais têm evidenciado que indivíduos que se utilizam mais da reavaliação do que da supressão revelam melhores indicadores afetivos, cognitivos, sociais e de bem-estar (Richards \& Gross, 2000).

O presente estudo identificou relações entre o uso das estratégias de reavaliação cognitiva e supressão emocional com indicadores sociodemográficos e, em especial com medidas afetivas. Os idosos viúvos, em média, relataram maior uso de reavaliação cognitiva, dado que encontra ressonância nos estudos de estresse e coping que também encontram maior uso de estratégias de reavaliação cognitiva ou de reinterpretação do significado de eventos estressantes. Segundo Aldwin (2007), a experiência da viuvez, por se referir em geral a um evento incontrolável, influencia o uso de modos indiretos ou de controle secundário para lidar com situações estressantes de vida.

Algumas correlações indicaram também a validade das medidas utilizadas no estudo. Destacam-se as correlações positivas de afetos positivos com satisfação com vida e as correlações negativas também dos afetos positivos com medidas de afetos negativos e depressão. Tais dados revelam que entre idosos os afetos positivos e negativos também revelam-se como construtos independentes, pois embora inversamente relacionados, essa relação se dá em diferentes proporções, ratificando os estudos a repeito da manutenção da diferenciação emocional na velhice (Ong $\&$ Bergeman, 2004).

Embora a estratégia de supressão emocional associese mais fortemente a indicadores negativos de saúde mental e adaptação (Richards \& Gross, 2000), os dados do presente estudo não evidenciaram essas relações de forma significativa, não revelando também associações com indicadores emocionais positivos (tais como afetos positivos e satisfação com a vida). O estudo compartilha com a perspectiva de Gross e John (2003) e John e Gross
(2004) de que as estratégias não são a priori essencialmente boas ou ruins, uma vez que dependem do contexto da experiência emocional e de variáveis desenvolvimentais atuantes, tais como os mecanismos de seleção, otimização e compensação cujo produto é uma resposta mais apropriada aos recursos emocionais disponíveis, o que não exclui a supressão emocional.

Entretanto, as estratégias de reavaliação cognitiva revelaram um maior número de correlações significativas que, em geral, associaram-se com melhores indicadores afetivos. Maior uso de reavaliação relacionou-se diretamente com experiência de mais afetos positivos e satisfação com a vida e indiretamente com afetos negativos $\mathrm{e}$ depressão. A força e direção das correlações entre reavaliação cognitiva e experiência de afetos positivos, afetos negativos e satisfação com a vida foram semelhantes às apresentadas por John e Gross (2004) em um quadro resumo dos achados de seus estudos anteriores com amostras jovens americanas.

Os dados coletados entre idosos também corroboram a concepção de que o maior uso da reavaliação cognitiva se relaciona com melhor saúde emocional e possa assim ser considerado, por exemplo, um fator protetor contra sintomas depressivos. Uma vez que esses sintomas, em geral, são disparados ou exacerbados por respostas negativas aos eventos estressantes de vida ou perdas, a reavaliação pode exercer proteção, evidenciando mais otimismo, autoestima e atuando na geração de estratégias criativas e adaptativas de enfrentamento do estresse.

Por outro lado, os dados a respeito das relações de supressão emocional com indicadores afetivos entre idosos não se comportaram como nos estudos com amostras mais jovens. Implicações de tais achados conduzem à concepção de que o construto de Regulação Emocional não se trata de um traço psicológico imutável, mas sensível ao processo de desenvolvimento e envelhecimento. Consistente com as teorias atuais sobre o envelhecimento emocional, os idosos relataram uma vida emocional predominantemente positiva e a capacidade preservada de regular as emoções de forma adaptativa.

Embora desenvolvido em um contexto estimulante e diferenciado como da UnATI - EACH e com uma amostra predominantemente feminina e saudável, o presente estudo contribui com a literatura nacional de pesquisa a respeito da regulação emocional entre idosos. Ao corroborar os achados internacionais sobre a temática, contribui ainda por derivar indicadores de validade e fidedignidade de um instrumento que visa medir o construto de Regulação emocional quando aplicado a indivíduos idosos.

Sugere-se que futuros estudos ampliem a investigação sobre a temática utilizando metodologias mais refinadas de análise de dados e desenhos longitudinais a fim de elucidar as relações entre regulação emocional e envelhecimento. Importante também incluir amostras comunitárias que abranjam a heterogeneidade dos idosos, identificando não só fatores relacionados ao comportamento psicométrico do instrumento (QRE), mas também as re- 
lações entre regulação emocional e indicadores mais amplos de adaptação na velhice, tais como variáveis cognitivas, sociais e saúde física e mental.

\section{Referências}

Aldwin, C. M. (2007). Stress, coping and development: An integrative perspective. New York: Guilford Press.

Almeida, O. P., \& Almeida, S. A. (1999). Confiabilidade da versão brasileira da Escala de Depressão em Geriatria (GDS) versão reduzida. Arquivos de Neuro-Psiquiatria, 57(2B), 421-426.

Blanchard-Fields, F., Stein, R., \& Watson, T. L. (2004). Age differences in emotion-regulation strategies in handling everyday problems. Journals of Gerontology: Psychological Sciences, 59(6), P261-P269.

Boian, A. C., Soares, D. S. M., \& Silva, J. (2009). Questionário de Regulação Emocional adaptado para a população brasileira. Retrieved December 15, 2010, from http:// spl.stanford.edu/pdfs/erq_portuguese_brazilian.pdf

Carstensen, L. L., Isaacowitz, D. M., \& Charles, S. T. (1999). Taking time seriously. A theory of socioemotional selectivity. American Psychologist, 54(3), 165-181.

Ehring, T., Tuschen-Caffier, B., Schnülle, J., Fischer, S., \& Gross, J. J. (2010). Emotion regulation and vulnerability to depression: Spontaneous versus instructed use of emotion suppression and reappraisal. Emotion, 10, 563-572.

Frank, M. H., \& Rodrigues, N. L. (2006). Depressão, ansiedade, outros distúrbios afetivos e suicídio. In E. V. Freitas, L. Py, A. L. Neri, F. A. X. C. Cançado, M. L. Gorzoni, J. Doll, Tratado de Geriatria e Gerontologia (2. ed., pp. 376-387). Rio de Janeiro, RJ: Guanabara Koogan.

Freire, S. A. (2001). Bem-estar subjetivo e metas de vida: Um estudo transversal com homens e mulheres pertencentes a três faixas de idade. (Tese de Doutorado não-publicada). Faculdade de Educação, Universidade Estadual de Campinas, SP.

Gillanders, S., Wild, M., Deighan, C., \& Gillanders, D. (2008). Emotion regulation, affect, psychosocial functioning, and well-being in hemodialysis patients. American Journal of Kidney Disease, 51(4), 651-662.

Gross, J. J. (1998a). Antecedent- and response-focused emotion regulation: Divergent consequences for experience, expression, and physiology. Journal of Personality and Social Psychology, 74, 224-237.

Gross, J. J. (1998b). The emerging field of emotion regulation: An integrative review. Review of General Psychology, 2, 271 299.

Gross, J. J., Carstensen, L. L., Pasupathi, M., Tsai, J., Gottestam, K., \& Hsu, A. Y. C. (1997). Emotion and aging: Experience, expression, and control. Psychology and Aging, 12, 590-599.

Gross, J. J., \& John, O. P. (2003). Individual differences in two emotion regulation processes: Implications for affect, relationships, and well-being. Journal of Personality and Social Psychology, 85, 348-362.

Gross, J. J., \& Levenson, R. W. (1993). Emotional suppression: Physiology, self-report, and expressive behavior. Journal of Personality and Social Psychology, 64, 970-986.

Hripcsak, G., \& Heitjan, D. F. (2002). Measuring agreement in medical informatics reliability studies. Journal of Biomedical Informatics, 35(2), 99-110.

John, O. P., \& Gross, J. J. (2004). Healthy and unhealthy emotion regulation: Personality processes, individual differences, and life span development. Journal of Personality, 72, 1301-1333.
John, O. P., \& Gross, J. J. (2007). Individual differences in emotion regulation strategies: Links to global trait, dynamic, and social cognitive constructs. In J. J. Gross (Ed.), Handbook of emotion regulation (pp. 351-372). New York: Guilford Press.

Kennedy, Q., Mather, M., \& Carstensen, L. L. (2004). The role of motivation in the age-related positivity effect in autobiographical memory. Psychological Science, 15, 208-214.

Lawton, M. P., Kleban, M. H., Rajagopal, D., \& Dean, J. (1992). Dimensions of affective experience in three age groups. Psychology and Aging, 7(2), 171-184.

Lazarus, R. S., \& Folkman, S. (1984). Stress, appraisal, and coping. New York: Springer.

Neri, A. L. (2002). Bienestar subjetivo en la vida adulta y en la vejez: hacia una Psicología positiva en la América Latina. Revista Latinoamericana de Psicologia, 34(2), 55-74.

Ong, A. D., \& Bergeman, C. S. (2004). The complexity of emotions in later life. Journals of Gerontology: Psychological Science, 59B, P117-P122.

Richards, J. M., \& Gross, J. J. (2000). Emotion regulation and memory: The cognitive costs of keeping one's cool. Journal of Personality and Social Psychology, 79(3), 410-424.

Santos, A. T. (2003). Controle percebido, senso de auto-eficácia e satisfação com a vida: Um estudo comparativo entre homens e mulheres pertencentes a três grupos de idade. (Tese de Doutorado não-publicada). Faculdade de Educação, Universidade Estadual de Campinas, SP.

Scheibe, S., \& Carstensen, L. L. (2010). Emotional aging: Recent findings and future trends. Journals of Gerontology: Psychological Sciences, 65B(2), 135-144.

Sheldon, K. M., Ryan, R. M., Rawsthorne, L. J., \& Ilardi, B. (1997). Trait self and true self: Cross-role variation in the big-five personality traits and its relations with psychological authenticity and subjective well-being. Journal of Personality and Social Psychology, 73(6), 1380-1393.

Siqueira, M. M. M., Martins, M. C. F., \& Moura, O. I. (1999). Construção e validação fatorial da EAPN: Escala de Ânimo Positivo e Negativo. Revista da Sociedade de Psicologia do Triângulo Mineiro, 2(3), 34-40.

Tamir, M., John, O. P., Srivastava, S., \& Gross, J. J. (2007). Implicit theories of emotion: Affective and social outcomes across a major life transition. Journal of Personality and Social Psychology, 92, 731-744.

Urry, H. L., \& Gross, J. J. (2010). Emotional regulation in old age. Current Directions in Psychological Science, 19, 352357.

Watson, D., Clark, L. A., \& Tellegen, A. (1988). Development and validation of brief measures of positive and negative affect: The PANAS Scales. Journal of Personality and Social Psychology, 54, 1063-1070.

Yahyagil, M. Y., \& Ýkier, S. (2009). Job satisfaction, emotion regulation, and stress relations and aging. Öneri Dergisi (Istanbul), 8(31), 43-51.

Yesavage, J. A., Brink, T. L., Rose, T. L., Lum, O., Huang, V., Adey, M., et al. (1983). Development and validation of a Geriatric Depression Screening Scale: A preliminary report. Journal of Psychiatric Research, 17, 37-49. 